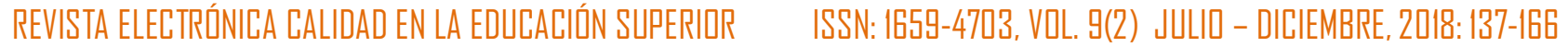

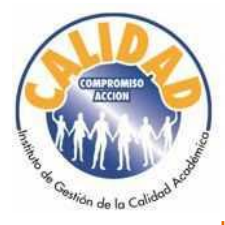

Vicerrectoría Académica

Instituto de Gestión de la Calidad Académica

http://investiga.uned.ac.cr/revistas/index.php/revistacalidad

Co-creando Excelencia

Corren electrónica: revistacalidad国uned.ac.cr

Estrategias pedagógicas utilizadas para la enseñanza de las ciencias del movimiento humano y la percepción estudiantil acerca de su impacto en el aprendizaje

Pedagogical strategies used for the teaching of human movement sciences and student perception about its impact on learning

Jorge E. Salas-Cabrera1, josaca10@hotmail.com

Carlos Álvarez-Bogantes², ceab.03@gmail.com

Stephanie Fabiola Ureña-Arias ${ }^{3}$, Sfabiola1995@gmail.com

Oscar José Guillen-Vargas ${ }^{4}$, Oscarguillen216@gmail.com

Universidad Nacional, Costa Rica

\title{
DOI: http://dx.doi.org/10.22458/caes.v9i2.2147
}

Volumen 9, Número 2

30 de noviembre de 2018

pp. $137-166$

Recibido: 30 de enero de 2018

Aprobado: 31 de agosto de 2018

\footnotetext{
${ }^{1}$ Ph.D. Coordinador Proyecto Rehabilitación Cardíaca Fase III, Académico de CIEMHCAVI, Universidad Nacional, Costa Rica. Correo electrónico: josaca10@hotmail.com

2 Ph.D. Coordinador Red Centroamericana de Investigación en Niñez y Calidad de Vida. Correo electrónico: ceab.03@gmail.com

${ }^{3}$ Estudiante Promoción de la Salud Física, Universidad Nacional (UNA), Costa Rica. Correo electrónico: sfabiola1995@gmail.com

${ }^{4}$ Estudiante Promoción de la Salud Física, Universidad Nacional (UNA), Costa Rica. Correo electrónico: oscarguillen216@gmail.com
}

Estrategias pedagógicas utilizadlas para la enseñanza de las ciencias del movimiento humano y la percepción estudiantil acerca de su impacto en el aprendizaje

Jorge E. Salas-Cabrera, Carlos Álvarez-Bogantes

Stephanie Fabiola Ureña-Arias, Oscar José Guillen-Vargas

DOI: http://dx.doi.org/10.22458/caes.v9i2.2147

\section{(c) (1) (5)}




\section{Resumen}

El propósito de esta investigación fue analizar las estrategias pedagógicas utilizadas para la enseñanza de las ciencias del movimiento humano y su impacto en el aprendizaje de estudiantes de educación superior. Se diseñó un estudio de corte cualitativo, con un grupo focal, observación participante y un cuestionario de evaluación docente. Participaron 32 estudiantes de 2 universidades públicas y 2 privadas; también participaron 12 docentes entre las cuatro universidades. La conclusión es que la mayoría de los profesores fueron percibidos como netos transmisores de información; el ambiente de clase fue percibido como superficial y poco significativo. Por otro lado, se demostró que en el caso de aquellos docentes que mostraron interés, respeto y preocupación por sus alumnos, su clase fue considerada significativa para aprender.

Palabras clave: estrategias pedagógicas, aprendizaje, movimiento humano

\section{Abstract}

The purpose of this research was to analyze the pedagogical strategies used for the teaching of human movement sciences and their impact on the learning of higher education students. A qualitative study was designed, with focus group, participant observation and teacher evaluation questionnaire. Participants were 32 students from 2 public and 2 private universities; 12 teachers from the four universities also participated. The conclusion is that most of the teachers were perceived by the students as clearly transmitters of information with class environments superficial and not significant. On the other hand, it was shown that those teachers, who showed interest, respect and concern for their students, the class was considered significant to learn.

Keywords: pedagogical strategies, learning, human movement

Estrategias pedagógicas utilizadas para la enseñanza de las ciencias del movimiento humano y la percepción estudiantil acerca de su impacto en el aprendizaje

Jorge E. Salas-Cabrera, Carlos Álvarez-Bogantes, 


\section{Introducción}

En las últimas décadas, la educación superior ha sufrido transformaciones en cuanto a los modelos pedagógicos, los cuales buscan desarrollar un mejor aprendizaje para el estudiante. Durante los procesos actuales de enseñanza-aprendizaje intervienen un sinnúmero de factores que buscan, a través de metodologías pedagógicas, generar un aprendizaje significativo que fortalezca la formación profesional de los estudiantes. Es claro que el método de enseñanza de los profesores tiene un impacto en el aprendizaje de los estudiantes de forma negativa o positiva (Torres, Ardisana, Hernández, Cabañas, 2006; Gargallo, 2008; Briceño, 2008).

En este sentido, durante las prácticas pedagógicas se presta atención a la forma en ¿qué? y ¿para qué? aprende una persona y se ignora el ¿cómo? Se presenta así, una relación interesante que genera cierta controversia acerca de si es la pedagogía centrada en la enseñanza la que provoca cambios en el comportamiento de la persona. Para Reigeluth (1983), la enseñanza como tal es de naturaleza prescriptiva, se centra en métodos de instrucción ( $\mathrm{y}$, por lo tanto, en la tarea docente), y el aprendizaje se acentúa desde su carácter descriptivo, en aspectos del proceso en el cual el protagonista es el discente. Para Martínez (s.f.), el proceso de aprendizaje es la modificación en la comprensión de un determinado contenido que presenta la persona y que no es atribuible al simple proceso de desarrollo (maduración), que no siempre es observable, y se fundamenta en las estrategias metodológicas y con la globalización de los resultados.

De acuerdo con lo anterior, las investigaciones ponen de manifiesto dos grandes modelos educativos: aquel que está basado explícitamente en la enseñanza y el que se enfoca en aspectos relacionados al aprendizaje (Molereo, 2003; Biggs, 2005, Alvarado, 2005). Desde la

Estrategias pedagógicas utilizadas para la enseñanza de las ciencias del movimiento humano y la percepción estudiantil acerca de su impacto en el aprendizaje

Jorge E. Salas-Cabrera, Carlos Álvarez-Bogantes,

Stephanie Fabiola Ureña-Arias, Oscar José Guillen-Vargas

DOI: http://dx.doi.org/10.22458/caes.v9i2.2147

(c) (i) (8) 
posición centrada en la enseñanza, lo que se busca es básicamente la reproducción de datos, toda la responsabilidad de generar la información que posteriormente será reproducida por el estudiante recae directamente en el profesor. No son importantes las creencias, opiniones o inquietudes de los estudiantes y los aspectos motivacionales los ejerce única y exclusivamente el docente. Por su parte, el modelo educativo enfocado en el aprendizaje centra su atención en el cambio mental, lo cual determina que lo aprendido es para la vida y para interpretar la realidad que lo rodea. La responsabilidad, en el modelo basado en el aprendizaje, se distribuye entre el docente y el estudiante, las ideas y creencias del estudiante son utilizadas para modificarlas conceptualmente y así prevenir errores (Gargallo, 2008; Adell, 2010).

Un estudio realizado por Gow y Kember (1993) mostró que aquellos estudiantes que se encontraban bajo estrategias pedagógicas relacionadas solamente a transmisión de información, respondieron con un aprendizaje de tipo superficial, sin embargo, aquellos estudiantes que estuvieron bajo el enfoque de estrategias pedagógicas relacionadas con la facilitación del aprendizaje, mostraron un manejo profundo de los contenidos.

Un estudio realizado por Lee, Srinivasan, Trail, Lewis, López (2011) evaluó el apoyo para el aprendizaje del estudiante como elemento clave en la optimización de las experiencias de cualquier entorno educativo; los resultados encontrados determinaron que utilizando cualquier modelo, si los estudiantes reciben el apoyo emocional, este tiene relación significativa con la satisfacción total del modelo de aprendizaje.

Los hallazgos de este estudio recomiendan a los docentes generar apoyo durante las prácticas educativas y proporcionarlas de una manera fácil. De acuerdo con Alterio y Pérez (2009), “...la función del docente según su desempeño, plantea la calidad de la docencia y

Estrategias pedagógicas utilizadas para la enseñanza de las ciencias del movimiento humano y la percepción estudiantil acerca de su impacto en el aprendizaje

Jorge E. Salas-Cabrera, Carlos Álvarez-Bogantes,

Stephanie Fabiola Ureña-Arias, Oscar José Guillen-Vargas

DOI: http://dx.doi.org/10.22458/caes.v9i2.2147

(c) (i) (8) 
está muy asociado a los procesos y resultados del desarrollo educativo, con lo que garantiza el perfeccionamiento de competencias formativas en el profesional...".

Bajo esta perspectiva, nace el siguiente cuestionamiento: ¿cuáles son las estrategias pedagógicas utilizadas por las y los académicos durante las sesiones presenciales para la enseñanza de las ciencias del movimiento humano? El propósito de esta investigación es conocer la percepción de los estudiantes en cuanto a las metodologías utilizadas por los docentes de educación superior y cómo las mismas pueden provocar un aprendizaje concreto que les facilite el desenvolvimiento en el campo laboral.

\section{METODOLOGÍA}

Desde el enfoque cualitativo, las técnicas de recolección de información se centran en la comprensión de una realidad que es considerada particular, como parte de un proceso histórico de construcción y vista a partir de la lógica y el sentir de sus protagonistas, es decir, desde una perspectiva interna (subjetiva) (Pérez, 2001).

\section{Participantes}

Para efectos de esta investigación, se contará con la participación de 32 estudiantes, los cuales se distribuirán de la siguiente forma: 8 estudiantes por centro superior de educación, entre hombres y mujeres, con edades entre los 21 y 30 años; deben haber cursado al menos un año de carrera. Además, se contará con la participación de 12 académicos, distribuidos en 3 académicos por institución, entre hombres y mujeres, con edades entre los 25 y 55 años, y con más de 3 años de estar ejerciendo la profesión.

\section{Instrumentos}

Los instrumentos utilizados para la recolección de datos fueron:

Estrategias pedagógicas utilizadas para la enseñanza de las ciencias del movimiento humano y la percepción estudiantil acerca de su impacto en el aprendizaje

Jorge E. Salas-Cabrera, Carlos Álvarez-Bogantes,

Stephanie Fabiola Ureña-Arias, Oscar José Guillen-Vargas

DOI: http://dx.doi.org/10.22458/caes.v9i2.2147

(c) (i) (8) 
Observación participante: en primera instancia, una bitácora en donde se realizaron apuntes acerca de aquellos aspectos interesantes que el observador evaluó durante su presencia en el salón de clase; para tal efecto, se construyó la bitácora de manera que sirva de guía durante el tiempo que el investigador se encuentre en el salón de clase.

Entrevista semi-estructurada: este instrumento sirvió de guía para recolectar información del estudiantado, así como de los educadores participantes de la investigación. De acuerdo con los antecedentes revisados en la teoría y con lo observado en el salón de clases, se construyó un instrumento que sirvió de herramienta para conocer las percepciones de los estudiantes acerca de las estrategias desarrolladas en el salón de clase y cómo las mismas impactan en su aprendizaje, además, se elaboró otro instrumento para aplicarlo a los académicos participantes y conocer el porqué de la utilización de ciertas estrategias durante el desarrollo de su curso.

Finalmente, se aplicó el instrumento: Cuestionario de Evaluación Docente para Docentes y Estudiantes del Sistema Nacional de Educación Superior Tecnológica. La validación de criterio fue revisada y aprobada por directivos del sistema, directores, subdirectores, jefes de departamento, profesores y alumnos en la ciudad de México (s.f.).

\section{Procedimiento}

Primeramente, se realizó un contacto con cada uno de los directores de las instituciones de educación superior, específicamente de las escuelas que desarrollan la carrera de movimiento humano. Posteriormente, se seleccionó aleatoriamente a los profesores de las distintas escuelas, siguiendo los criterios establecidos para el personal docente como: edad, tiempo de trabajo y que fueran parte del cuerpo docente. Cada uno de los docentes llenó un consentimiento informado de participación, seguido de la solicitud de poder asistir a dos

Estrategias pedagógicas utilizadas para la enseñanza de las ciencias del movimiento humano y la percepción estudiantil acerca de su impacto en el aprendizaje

Jorge E. Salas-Cabrera, Carlos Álvarez-Bogantes,

Stephanie Fabiola Ureña-Arias, Oscar José Guillen-Vargas

DOI: http://dx.doi.org/10.22458/caes.v9i2.2147

\section{(c) (i) (8)}


clases suyas para realizar la observación participante. Una vez concluida la fase anterior, se realizó la entrevista semi-estructurada a los 8 estudiantes participantes del curso, la cual fue grabada en una grabadora de audio para recolectar toda la información necesaria. Finalmente, a este grupo de estudiantes se les aplicó el cuestionario y a los profesores observados se les hizo una pequeña entrevista.

\section{Análisis de datos}

La triangulación de métodos es la combinación de fuentes de datos y métodos de investigación en el estudio de un fenómeno singular. Para el análisis de datos se realizó una triangulación, estableciéndose las categorías de análisis, las cuales permitieron realizar codificaciones de la información que se generó para, posteriormente, desarrollar el análisis de interpretación. Dicho análisis estuvo basado en los insumos facilitados por los docentes, estudiantes y por el cuestionario de evaluación del desempeño docente. Una vez que se obtuvo el análisis correspondiente, se construyó un texto, de manera narrativa, con los principales hallazgos alcanzados por la investigación.

\section{Resultados}

A continuación, se presentan en este apartado los resultados obtenidos en las diferentes entrevistas. En cada tabla se presentan las expresiones que más se repitieron entre los estudiantes y entre los docentes. Cada tabla representa las percepciones de los participantes acerca de las grandes categorías de análisis escogidas por el grupo investigador.

Tabla 1: Información presentada por el observador acerca de las estrategias pedagógicas, motivación y comprensión de contenidos que los docentes de los centros de educación superior públicos y privados desarrollan en la carrera de Educación Física.

\section{CATEGORÍA}

\section{CATEGORÍA}

Estrategias pedagógicas utilizadas para la enseñanza de las ciencias del movimiento humano y la percepción estudiantil acerca de su impacto en el aprendizaje

Jorge E. Salas-Cabrera, Carlos Álvarez-Bogantes,

Stephanie Fabiola Ureña-Arias, Oscar José Guillen-Vargas

DOI: http://dx.doi.org/10.22458/caes.v9i2.2147

(c) (i) (8)

Artículo protegido por licencia Creative Commons 
- Algunos estudiantes ponen atención, otros están en sus cosas.

- Estudiantes con maletines en la mesa, tipo almohada.

- Algunos estudiantes están en la computadora, celular y dormidos.

- El profesor motiva, más allá de los contenidos, a marcar diferencia afuera.

- El profesor se mantiene cerca para así evacuar cualquier duda.

- Utiliza preguntas en el ambiente para que los estudiantes respondan.

- Usa ejemplos de la vida real para compresión, el profesor relaciona contenidos de otras clases para integrar.

- Utiliza imágenes dentro de la presentación para lograr una mejor comprensión.

- Exposición PPT muy cargada de letras.

- El profesor permanece sentado en el escritorio, no tiene visualización del grupo y explica desde el escritorio.

- Inicia con charla magistral, usa el vídeo beam (PPT) y la pizarra para explicar los contenidos.

- Empieza la exposición de los estudiantes y la profesora se sienta al frente, dando la espalda al grupo, no sabe lo que pasa atrás.

- La clase es práctica, un caso hipotético donde los estudiantes deben atender la situación, el profesor va guiando el proceso.

- Realiza práctica para examen y explica cómo será la evaluación de la próxima semana.

- El profesor se ubica al frente y utiliza un silbato para dar las órdenes y con una pizarra explica propiamente el contenido.

\section{UNIVERSIDADES DEL SECTOR PRIVADO}

- Los de atrás se recuestan en el escritorio.

- Dos alumnos completamente dormidos.

- El profesor continúa explicando y, de vez en cuando, utiliza palabras motivadoras para con sus estudiantes.
- Proyecta un documento en PDF del Ministerio de Educación, el cual no se puede leer ya que las letras son muy pequeñas, la vista en proyección se ve borrosa.

- Comienza a leer textual el documento, siempre sentado en su silla, sin moverse de ahí.

- Se pone de pie al lado del escritorio, explica viendo hacia abajo, repite para los estudiantes

Estrategias pedagógicas utilizadas para la enseñanza de las ciencias del movimiento humano y la percepción estudiantil acerca de su impacto en el aprendizaje

Jorge E. Salas-Cabrera, Carlos Álvarez-Bogantes, 
- Estudiantes utilizan los celulares y no ponen atención. que no entendieron.

- El profesor explica la clase con un PPT, apaga la luz para ver mejor, el profesor realiza una clase magistral sobre aspectos de táctica y estrategia.

- La presentación en PPT del profesor está muy cargada de palabras.

Observaciones: en la tabla 1 se presentan los resultados obtenidos durante la observación realizada a cada uno de los docentes y las estrategias pedagógicas utilizadas durante el desarrollo de un contenido en específico; esta observación se realizó en las cuatro instituciones que participaron en la investigación. Los resultados son presentados bajo el lineamiento de cuatro grandes categorías de análisis. En la primera categoría se presenta la motivación, como se puede apreciar, mucho de lo que los docentes desarrollan en cada contenido de curso es tedioso y poco motivador, los estudiantes pierden el deseo por aprender y, más bien, llegan a los salones de clase a dormir.

Pareciera ser que a los docentes poco les es llamativo a la hora de escoger la estrategia pedagógica para enseñar los contenidos de los cursos y, además, pareciera que las metodologías para generar motivación, tanto en las instituciones públicas como privadas, no es la más conveniente. Este aspecto es de gran relevancia durante la enseñanza académica, pues el generar motivación provoca una emoción positiva y la posibilidad de un aprendizaje significativo a largo plazo. En este sentido, Ratelle, Guay, Vallerand, Larose, \& Senécal (2007) expresan que los alumnos con perfiles de motivación elevados presentan mejores calificaciones y continúan sus estudios, mientras que aquellos estudiantes poco motivados tienden a abandonarlos. Además, hay que prestar especial atención al uso de sus celulares, los cuales son elementos distractores, si no son utilizados como una herramienta didáctica.

Estrategias pedagógicas utilizadas para la enseñanza de las ciencias del movimiento humano y la percepción estudiantil acerca de su impacto en el aprendizaje

Jorge E. Salas-Cabrera, Carlos Álvarez-Bogantes,

Stephanie Fabiola Ureña-Arias, Oscar José Guillen-Vargas

DOI: http://dx.doi.org/10.22458/caes.v9i2.2147

\section{(c) (i) $\$$}


Por su parte, en las estrategias pedagógicas se puede observar que se siguen presentando las metodologías de años atrás, con la variante de que se usan las nuevas tecnologías como herramientas para el proceso de enseñanza-aprendizaje, como es el caso de Power Point (PPT). Sin embargo, durante el desarrollo de las clases se siguen realizando charlas magistrales; las presentaciones están muy cargadas de información; durante las clases de noche se apaga la luz, lo que deja al aula totalmente oscura; y la poca movilidad de parte del docente dentro del salón de clase provoca una baja atención en los contenidos que se están desarrollando. Es indispensable valorar la posibilidad de realizar cambios en la metodología de enseñanza, generar en los estudiantes el papel dinámico en la construcción de su propio conocimiento y brindar a los docentes la mediación que facilite la transformación del estudiante de un ser pasivo a uno activo. De acuerdo con Rivero, Bernal, Santana, Pedraza (2014), la enseñanza de contenidos está dejando de tener carácter primordial en el proceso de enseñanza-aprendizaje universitario, es indispensable que los profesores enseñen estrategias de aprendizaje que les permitan a los estudiantes lograr la mayor independencia cognoscitiva y autonomía posibles, bajo la guía del profesor.

Tabla 2: Información presentada por el observador acerca de los procesos de retroalimentación y comprensión de contenidos que los docentes de los centros de educación superior públicos y privados desarrollan en la carrera de Educación Física.

\section{CATEGORÍA RETROALIMENTACIÓN \\ CATEGORÍA COMPRENSIÓN DE CONTENIDOS UNIVERSIDADES DEL SECTOR PÚBLICO}

- Pocas preguntas, sin embargo, se da proceso de intercambio de opiniones en la clase.

- Estudiantes preguntan sobre la charla, la explicación es muy rápida por parte del profesor.
- El profesor utiliza bromas con los estudiantes para explicar los contenidos, de manera que estos queden claros.

- Da muchos ejemplos para reforzar el contenido.

- El profesor introduce algunos ejemplos en

Estrategias pedagógicas utilizadas para la enseñanza de las ciencias del movimiento humano y la percepción estudiantil acerca de su impacto en el aprendizaje

Jorge E. Salas-Cabrera, Carlos Álvarez-Bogantes,

Stephanie Fabiola Ureña-Arias, Oscar José Guillen-Vargas

DOI: http://dx.doi.org/10.22458/caes.v9i2.2147

\section{(C) (1) $(9$}


- La profesora pregunta si alguien tiene preguntas, ningún estudiante responde, ella lanza preguntas, nadie las contesta, ella misma da la respuesta.

- El profesor siempre corrige errores. competencia para dejar más claro el contenido.

- El profesor explica cómo se va a desarrollar la clase.

- Los estudiantes se distraen por la ventana, ya que cortan un árbol, muchos se asoman.

\section{UNIVERSIDADES DEL SECTOR PRIVADO}

- Lanza preguntas, nadie contesta.

- Una estudiante le pregunta acerca del tema y la respuesta se la da leyendo textual el documento en PDF.

- El profesor lanza una pregunta y espera respuestas de los alumnos.

- Algunos estudiantes preguntan.
- Los estudiantes comprenden la dinámica del curso y participan ampliamente.

- El profesor pregunta si entendieron.

- Otro estudiante comenta sobre lo que explica el profesor en la pizarra.

- Da muchos ejemplos para reforzar el contenido.

- Los estudiantes disfrutan del juego y aprenden.

Observaciones: para el caso de la categoría de retroalimentación, se aprecia que en ambos tipos de instituciones, tanto públicas como privadas, los docentes realizan las mismas estrategias pedagógicas: realizar preguntas a los grupos acerca de los contenidos que se están analizando, sin embargo, los estudiantes no responden. Es claro que la posibilidad de equivocarse hace que los alumnos esperen que sea otro compañero quien se aventure a dar una posible respuesta, esto va muy acorde con el aprendizaje reflexivo, en el que se manifiesta que los estudiantes reflexionan acerca de la nueva información y se toman un tiempo antes de dar una respuesta, que en muchos casos no es expresada por temor a equivocarse.

De acuerdo con Craveri y Anido (2008), los estudiantes de educación superior prefieren inicialmente el estilo de aprendizaje reflexivo, sin implicar que este estilo de aprendizaje incida sobre el rendimiento académico Las tendencias pueden variar entre sí, es posible

Estrategias pedagógicas utilizadas para la enseñanza de las ciencias del movimiento humano y la percepción estudiantil acerca de su impacto en el aprendizaje

Jorge E. Salas-Cabrera, Carlos Álvarez-Bogantes,

Stephanie Fabiola Ureña-Arias, Oscar José Guillen-Vargas

DOI: http://dx.doi.org/10.22458/caes.v9i2.2147

(c) (i) (8) 
que la influencia entre estilo y rendimiento esté determinada por la estrategia de formación o enseñanza empleada por el docente.

Para la categoría de comprensión de contenidos, se aprecia que los profesores utilizan estrategias idóneas para el entendimiento de los contenidos por parte de los estudiantes; muchos de ellos realizan comparaciones del nuevo conocimiento con situaciones de la vida real, además, emplean ejemplos para reforzar lo que están explicando y que, de esta manera, quede más claro. En ese sentido, Tolosa y Zerpa (2009) explican que las estrategias cognitivas y meta-cognitivas de aprendizaje han demostrado ser eficientes en el desarrollo de las competencias de aprendizaje activo, por lo que es recomendable concentrase en aspectos que tengan que ver con una adecuada comprensión de los contenidos que se explican durante las lecciones: preguntando, ejemplificando y volviendo a analizar lo ya explicado, de manera que se interiorice lo aprendido de manera significativa. Finalmente, algunos de estos docentes inician la clase explicando lo que se va a realizar durante toda la lección, de manera que los estudiantes conocen con antelación lo que se va a desarrollar en clase.

Tabla 3: Percepciones que más coincidían en las categorías de motivación y estrategias pedagógicas que manifestaron los estudiantes de los centros de educación superior públicos y privados en donde se imparte la carrera de Educación Física.

\begin{tabular}{|c|c|}
\hline $\begin{array}{l}\text { CATEGORÍA } \\
\text { MOTIVACIÓN }\end{array}$ & $\begin{array}{c}\text { CATEGORÍA } \\
\text { ESTRATEGIAS PEDAGÓGICAS }\end{array}$ \\
\hline \multicolumn{2}{|c|}{ UNIVERSIDADES DEL SECTOR PÚBLICO } \\
\hline $\begin{array}{l}\text { - “...Hay momentos en los que digo, } \\
\text { di no, ya resígnese, no va a } \\
\text { entender nada". } \\
\text { - "...Un día a mí me sucedió que me }\end{array}$ & $\begin{array}{l}\text { - "...A nosotros no nos gusta estar sentados } \\
\text { cuatro horas viendo a una persona ahí } \\
\text { exponiendo..." } \\
\text {-...A mí de nada me sirve ver la teoría del } \\
\text { movimiento, si en realidad yo tengo que ir a }\end{array}$ \\
\hline
\end{tabular}

Estrategias pedagógicas utilizadas para la enseñanza de las ciencias del movimiento humano y la percepción estudiantil acerca de su impacto en el aprendizaje

Jorge E. Salas-Cabrera, Carlos Álvarez-Bogantes,

Stephanie Fabiola Ureña-Arias, Oscar José Guillen-Vargas

DOI: http://dx.doi.org/10.22458/caes.v9i2.2147

(c) (i) (-)

Artículo protegido por licencia Creative Commons 
bajaban las lágrimas porque yo decía: no me puedo dormir..."

- "...Él dice: laboratorio, ya vamos para el laboratorio, yo totalmente me despierto, son momentos de lucidez..."

- “...A veces los profesores como que nos llenan de teoría..."

- "...Pero falta algo que motive a que todos quieran aprender, que todos participen, que todos quieran intentar hacerlo..." aprender cómo se hace..."

- "...Las evaluaciones deben asemejarse a lo que se va a hacer a la hora laboral..."

- "...Él lo explica como si fuera entrenador y nosotros fuéramos a ser atletas, no estudiantes o futuros profesores..."

- "...Si el profe primero me enseñara la prueba y luego hablamos de toda la teoría, entonces yo puedo decir: ah bueno, sí, esto se aplica así, pero antes no, estoy bateada. En los exámenes abarca mucha materia y uno ya no se acuerda..."

- "...Le hace falta saber cómo llegarles a los estudiantes porque a veces se estresa muy fácil y ya se le hace un mundo todo y al final nadie termina haciendo nada..."

- "...Las explicaciones, se le complica y no tiene mayor culpa, no a todos se les da eso..."

\section{UNIVERSIDADES DEL SECTOR PRIVADO}

- "...Sí nos mantiene motivados, porque crea un ambiente de camaradería..."

- "...Motiva mucho, ya que siempre nos habla de: cuando ustedes sean profesores o educadores, qué harían, nos da consejos y se puede aplicar a la vida real..."

- "...Todo depende de la materia que se esté dando..."

- "...Hay profesores que saben muchísimo pero no saben transmitir la información..."

- "...Él motiva al $100 \%$ en las clases, dan ganas de ir siempre, en comparación a otras que son tediosas y aburridas..."
- "...Después de la clase siempre se hace una actividad como de reforzamiento, con eso aprendemos un montón..."

- "...El profe nos enseña haciendo, por ejemplo, cuando nos pintamos los músculos en clase yo me los aprendí todos..."

- "...Las clases magistrales son pesadas y aburridas..."

- "...La teoría en clase se complementa con la practica en el gimnasio y es ahí donde él enfatiza, capta nuestra atención, hace clases diferentes, nunca repite y eso hace que sea dinámico y llame la atención, tiene la chispa y si no nos queda algo claro, él retoma, es un profesor excelente..."

- "...Las evaluaciones van acordes con la temática..."

Estrategias pedagógicas utilizadas para la enseñanza de las ciencias del movimiento humano y la percepción estudiantil acerca de su impacto en el aprendizaje

Jorge E. Salas-Cabrera, Carlos Álvarez-Bogantes,

Stephanie Fabiola Ureña-Arias, Oscar José Guillen-Vargas

DOI: http://dx.doi.org/10.22458/caes.v9i2.2147

\section{(c) (i) (8)}


- “...Él explica lo que realmente importa, eso es mi parte favorita..."

- “...Y él se esfuerza por que uno aprenda, y hace que uno se motive, hace que uno quiera tomar apuntes."

“... las clases magistrales, cargadas de teoría dan sueño..."

“...falta más motivación por parte de los profesores a sus alumnos..."

“...a mí me gusta que él pone atención en lo que uno pregunta, si el profe está motivado nosotros también..."
• “...Él da ejemplos para poder entender...”

“... la teoría es necesaria, pero sin la práctica de nada nos sirve..."

“...necesitamos evaluaciones acordes al campo laboral, no nos ayuda que nos expliquen como si fuéramos atletas, vamos a ser profesores..."

"...no me gustan porque son charlas muy magistrales, podría ser un poco más dinámico..."

Observaciones: anteriormente se presentó la tabla 3, que resume aquellas percepciones más significativas y que se repitieron a lo largo de toda la investigación y en las diferentes instituciones que imparten la carrera de Educación Física en Costa Rica. Para la categoría de motivación, se puede observar que existen diversas opiniones en cuanto a la estrategia de motivación que utilizan los docentes, en la mayoría de las opiniones los estudiantes sienten que las clases son muy aburridas porque están cargadas de teoría y los docentes no realizan acciones diferentes para hacer de esa teoría algo que genere curiosidad y llame la atención del estudiantado. De acuerdo con Montico (2004), motivar al alumno en cada clase es tratar, en lo posible, de orientarlo en una dirección y buscar que se sigan los pasos necesarios para optimizar el vínculo entre la enseñanza y el aprendizaje.

Como se puede apreciar, poco se realiza en las instituciones públicas con respecto a generar ambientes que despierten la motivación del estudiantado, parece ser que los docentes se preocupan más por terminar contenidos que por generar procesos que coadyuven a una

Estrategias pedagógicas utilizadas para la enseñanza de las ciencias del movimiento humano y la percepción estudiantil acerca de su impacto en el aprendizaje

Jorge E. Salas-Cabrera, Carlos Álvarez-Bogantes,

Stephanie Fabiola Ureña-Arias, Oscar José Guillen-Vargas

DOI: http://dx.doi.org/10.22458/caes.v9i2.2147

(c)

Artículo protegido por licencia Creative Commons 
mejor comprensión de lo que se está impartiendo. Un estudio realizado por Standage, Duda y Ntoumanis (2005) demostró que la falta de motivación dentro de un salón de clase puede dar lugar a efectos negativos como la depresión, apatía, incompetencia, aburrimiento e infelicidad. Las estrategias pedagógicas implementadas por los docentes deben generar ambientes de emociones placenteras, de tal forma que incentiven a la participación activa de los estudiantes.

En el caso de las estrategias pedagógicas, pareciera ser que los docentes en las instituciones públicas se esfuerzan poco por generar metodologías que faciliten el aprendizaje del estudiante, la mayoría de los estudiantes manifiesta que las charlas magistrales siguen siendo estrategias pedagógicas poco llamativas y que, en definitiva, es en la parte práctica en donde se despierta su atención y se genera el aprendizaje. De acuerdo con De Miguel (2005), en los salones de clase de educación superior la práctica más habitual y característica en la enseñanza universitaria es la clase teórica, esta estrategia, por sí sola, no es muy recomendable para el generar un adecuado proceso de aprendizaje autónomo de los alumnos.

Por su parte, en las instituciones privadas los docentes tratan de generar estrategias que motiven la participación del estudiante, además, se les explica claramente el proceso de evaluación, la cual va de acuerdo con los contenidos del curso, se utilizan muchos ejemplos de vida que les permiten a los estudiantes comprender de manera clara lo que se les está enseñando.

En este sentido, De Miguel (2005) comenta que para lograr que el alumno sea el responsable y protagonista de su propio proceso de aprendizaje es necesario que tenga una participación activa en la organización y gestión de la propia actividad, es decir, su propio proceso de aprendizaje. Marrero y Santana (2016) explican que para un proceso exitoso en

Estrategias pedagógicas utilizadas para la enseñanza de las ciencias del movimiento humano y la percepción estudiantil acerca de su impacto en el aprendizaje

Jorge E. Salas-Cabrera, Carlos Álvarez-Bogantes,

Stephanie Fabiola Ureña-Arias, Oscar José Guillen-Vargas

DOI: http://dx.doi.org/10.22458/caes.v9i2.2147

(c) (i) (-) 
el aprendizaje es necesaria la disposición del personal docente hacia la modificación en las estrategias pedagógicas, lo cual constituye un eslabón fundamental para lograr una educación que garantice la adquisición de conocimientos.

Como se explicó, es indispensable realizar cambios en las estrategias pedagógicas de enseñanza. Los estudiantes han manifestado estar disconformes con las charlas magistrales que consumen mucho tiempo y que les generan aburrimiento y falta de atención, los docentes deben utilizar mayor cantidad de herramientas didácticas que despierten la curiosidad del estudiantado, de manera que el proceso de enseñanza y aprendizaje sea exitoso.

Tabla 4: Percepciones que más coincidían en las categorías de retroalimentación y comprensión de contenidos que manifestaron los estudiantes de los centros de educación superior públicos y privados en donde se imparte la carrera de Educación Física.

\section{CATEGORÍA RETROALIMENTACIÓN CATEGORIA UNIVERSIDADES DEL SECTOR PÚBLICO}

- “...Cuando estábamos viendo los sistemas energéticos oxidativos, el profe nos puso en grupos a decirnos en cuál deporte se usaba cada sistema. Ya cuando lo puse en práctica lo entendí, antes de eso no había entendido absolutamente nada..."

- "...Explica no solo en deporte sino en casos de la vida cotidiana que cualquier persona que no esté metida en esta carrera pueda entenderlo..."

- "...Esta carrera es aprender a
- “...Los laboratorios me han parecido muy buenos, a mí me han ayudado a entender muchas cosas..."

- "...Ha sido muy paciente, nos ha enseñado y explicado cómo hacer un laboratorio, las partes que tiene que llevar..."

- "...Él explica muy bien, conoce demasiado sobre el tema, y tiene la paciencia para ir explican paso por paso y eso es bueno..."

- "...Cuando se habla y no se ha visto lo que se va a hacer, sí cuesta más hacer la relación. Hasta que usted ya lo ve puede hacer la conexión, es mejor que se haga

Estrategias pedagógicas utilizadas para la enseñanza de las ciencias del movimiento humano y la percepción estudiantil acerca de su impacto en el aprendizaje

Jorge E. Salas-Cabrera, Carlos Álvarez-Bogantes,

Stephanie Fabiola Ureña-Arias, Oscar José Guillen-Vargas

DOI: http://dx.doi.org/10.22458/caes.v9i2.2147

(c) (i) (8) 
hacer, no a quedarse con la idea, es más significativa la práctica, yo me puedo acordar desde el primer día todo lo que hemos hecho..."

- "...Yo ignoro toda la parte teórica, no puedo concentrarme, hasta que ya lo pone en práctica ya puedo asimilarlo..."

- "...Él incentiva, cómo esto le va a ayudar cuando trabaje en tal lado..."

- "...Los laboratorios hechos como parte de la clase práctica fueron buenos y la profe los supo explicar bastante bien..."

- “...Se tenía muy claro cuáles eran los objetivos, en ningún momento improvisó..."

- "...Digamos, las estrategias de enseñanza se puede decir que están bien, nada más que él debería aprender cómo llegarles a los estudiantes..."

- "...Él lo explica una vez y listo, es cosa suya, él dice: ey gente, vamos a clase, y si la gente no hizo caso, él no va a estar de necio..." primero la evaluación y luego se hable de lo que pasó, ya uno le pone más atención y entiende más por qué..."

- “...La práctica la he podido transferir y he visto cambios beneficiosos en las áreas que yo doy..."

- "...Beneficia mucho con los quices..."

- "...Todo lo que vemos en la práctica se evalúa exactamente igual en el examen, entonces uno sabe lo que va a hacer en el examen y va a ir a hacer afuera..."

- "...Es muy bueno para nuestro aprendizaje, porque uno asocia con lo que ha vivido y con lo que puede vivir..."

- "...Él dejar que el estudiante entienda por sí solo lo que se ha explicado, eso ayuda..."

- "...Hace que uno sea el que tiene que buscar información, buscar las actividades y planificarlas le ayuda a uno en esa parte..."

\section{UNIVERSIDADES DEL SECTOR PRIVADO}

- “... Igual en la clase cada quien aporta sus experiencias de acuerdo a sus áreas de trabajo..."

- “...La parte de la práctica después de la teoría es fundamental, surgen dudas que cuando uno solo escucha la teoría no tiene, pero que cuando lo pone en práctica sí..."
- “...Más impacto en la parte práctica."

- "....Nos gusta aprender haciendo, porque a la hora de trabajar, hay que aplicar lo aprendido..."

- “...Sí he aprendido, pero porque lo he practicado, todo lo practicado lo recuerdo bien..."

- "...Yo siento que puedo decir firmemente

Estrategias pedagógicas utilizadlas para la enseñanza de las ciencias del movimiento humano y la percepción estudiantil acerca de su impacto en el aprendizaje

Jorge E. Salas-Cabrera, Carlos Álvarez-Bogantes,

Stephanie Fabiola Ureña-Arias, Oscar José Guillen-Vargas

DOI: http://dx.doi.org/10.22458/caes.v9i2.2147 
- "...La profe responde las preguntas y eso ayuda a entender mejor..."

- “...Él ayuda, si algo está mal, nos corrige y nos da otra oportunidad, es muy bueno..."

- “...Él nos incluye en la clase, nos pone a hablar, a dar ejemplos y él nos da ejemplos..." que con la clase práctica yo aprendo al $100 \% \ldots .$.

- "...todo lo que me enseña me sirve para trabajar..."

- “...Él nos prepara para los exámenes, para el futuro en la carrera. La evaluación que él hace son mejores que las de exámenes teóricos. Él nos enseña con casos de la vida real, ejemplos básicos que vemos todos los días. Nos pregunta y hace que participemos, nos da ejemplos de diferentes casos, nos hace pensar..."

“...para mí, la parte práctica de este curso es excelente, pero la teórica yo no me acuerdo de nada..."

"...hasta que se lleva a cabo la parte práctica, se logra comprender la teoría..."

“... cuando el profe está atento, corrige y ayuda, se logra un mejor entendimiento..." "...me ha parecido muy buen guía, él explica muy bien, conoce demasiado sobre el tema, y tiene la paciencia para ir explicando paso por paso ..."

"...nos transfiere a base de experiencias, entonces uno en la mente se va haciendo una mejor idea de cómo actuar en el campo, que es ya en el área donde vamos a trabajar..."

“... Puedo decir firmemente que con la clase práctica yo aprendo al $100 \%$, todo lo que me enseña me sirve..."

Observaciones: en la tabla 4 pueden apreciarse dos grandes categorías de análisis que son de mucha importancia para los cursos, estas están definidas, tanto por la retroalimentación como por la comprensión de contenidos. En la categoría de retroalimentación y comprensión de contenidos que realizan los docentes, los estudiantes perciben qué tanto, desde lo público como en lo privado, los docentes tratan de generar algún tipo de retroalimentación de los contenidos explicados para reforzar el aprendizaje y utilizar ejemplos de la vida real; sin embargo, los estudiantes de ambos tipos de instituciones siguen insistiendo en que la práctica en esta carrera es de suma importancia y que es a través de ella que comprenden

Estrategias pedagógicas utilizadas para la enseñanza de las ciencias del movimiento humano y la percepción estudiantil acerca de su impacto en el aprendizaje

Jorge E. Salas-Cabrera, Carlos Álvarez-Bogantes,

Stephanie Fabiola Ureña-Arias, Oscar José Guillen-Vargas

DOI: http://dx.doi.org/10.22458/caes.v9i2.2147

\section{(c) (i) $\$$}


mejor los contenidos que se les explica y que, de acuerdo con sus percepciones, existe muy poca práctica y esto provoca muy poco aprendizaje significativo.

Según Dewey (1997), al hacer, la persona aprende cosas sobre sí misma. En este sentido, es indispensable explicar los contenidos de un curso utilizando las prácticas como una herramienta didáctica que potencialice y genere un aprendizaje significativo en los estudiantes, sobre todo si se está ante una profesión que demanda mucho movimiento humano. Esta idea se refuerza de acuerdo con lo que comentan Boström, Josefsson (2006), en el sentido de que aprender haciendo provoca en el alumno un mayor aprendizaje, pues aplica y vive los conocimientos y aptitudes durante la práctica para después usarlos en la realidad. estudiantil acerca de su impacto en el aprendizaje

Jorge E. Salas-Cabrera, Carlos Álvarez-Bogantes, 
Tabla 5: Percepciones más importantes acerca de las categorías: motivación y estrategias pedagógicas que tienen los docentes de los centros de educación superior públicos y privados en donde se imparte la carrera de Educación Física.

\begin{tabular}{|c|c|}
\hline $\begin{array}{l}\text { CATEGORÍA } \\
\text { MOTIVACIÓN }\end{array}$ & $\begin{array}{r}\text { CATEGORÍA } \\
\text { ESTRATEGIAS PEDAG }\end{array}$ \\
\hline \multicolumn{2}{|c|}{ UNIVERSIDADES DEL SECTOR PÚBLICO } \\
\hline $\begin{array}{l}\text { - "... Hay que saber cómo motivar a } \\
\text { los alumnos, todos son diferentes, } \\
\text { para que le den importancia y } \\
\text { relevancia al tema que se está } \\
\text { explicando..." } \\
\text { "...Este es el deseo de todo } \\
\text { profesor, que sus estudiantes estén } \\
\text { motivados y así puedan entender y } \\
\text { aprovechar la información..." } \\
\text { - "...Más que la forma de aprender de } \\
\text { las personas es el interés de las } \\
\text { personas sobre lo que uno está } \\
\text { explicando..." } \\
\text { "...Y yo les digo: es muy fácil, aquí } \\
\text { nadie se queda, ponga atención al } \\
\text { curso y cuando ejecuten no van a } \\
\text { tener problema..." } \\
\text { ".... La motivación de ellos cambia de } \\
\text { la teoría a la práctica, siempre hay } \\
\text { un } 40 \% \text { que no hace nada y del } \\
60 \% \text { restante un } 20 \% \text { hace } \\
\text { preguntas y participa..." } \\
\text { ".... ta teoría no les gusta..." }\end{array}$ & $\begin{array}{l}\text { - “...Las estrategias son las correctas, esto se } \\
\text { comprueba por medio de las evaluaciones que } \\
\text { yo hago..." } \\
\text { - “...Dentro de lo que se ha desarrollado, pienso } \\
\text { que es adecuado, diverso y de buen gusto } \\
\text { para los estudiantes..." } \\
\text { - "...Son actividades diversas, no soy de los que } \\
\text { solo exámenes y quices, yo evalúo con } \\
\text { trabajos de campo, con películas..." } \\
\text { - “...Yo no soy un profesor de decirles: hagan, } \\
\text { yo hago con ellos..." } \\
\text { - “...yo utilizo varias estrategias de aprendizaje, } \\
\text { una de ellas es la estrategia de comando } \\
\text { directo..." } \\
\text { - "...en las actividades prácticas yo les entrego } \\
\text { indicaciones escritas y no me garantizo mucho } \\
\text { de yo explicarlas, parte de lo que yo quiero es } \\
\text { que ellos aprendan a interpretar..." }\end{array}$ \\
\hline \multicolumn{2}{|c|}{ UNIVERSIDADES DEL SECTOR PRIVADO } \\
\hline $\begin{array}{l}\text { - “...En los trabajos se les dice lo } \\
\text { negativo, pero también lo positivo } \\
\text { para hacerles ver los esfuerzos } \\
\text { buenos que ellos tienen..." } \\
\text { - "...Viene la parte de la vocación de } \\
\text { uno como docente, muchos }\end{array}$ & $\begin{array}{l}\text { - “...las estrategias siempre van enfocadas para } \\
\text { que los alumnos tengan un mejor } \\
\text { aprendizaje..." } \\
\text { - “...mis cursos se prestan mucho por la } \\
\text { temática a comparar con casos reales y sí } \\
\text { impactan mucho en los y las estudiantes, ya }\end{array}$ \\
\hline
\end{tabular}

Estrategias pedagógicas utilizadas para la enseñanza de las ciencias del movimiento humano y la percepción estudiantil acerca de su impacto en el aprendizaje

Jorge E. Salas-Cabrera, Carlos Álvarez-Bogantes,

Stephanie Fabiola Ureña-Arias, Oscar José Guillen-Vargas

DOI: http://dx.doi.org/10.22458/caes.v9i2.2147

\section{(c) $(1) \Theta$}




\begin{tabular}{|c|c|}
\hline $\begin{array}{l}\text { estudiantes no tienen esa vocación, } \\
\text { yo hago mucho hincapié en eso..." } \\
\text { - "...Que ellos aprovechen lo que uno } \\
\text { les está transmitiendo, pero si no } \\
\text { tienen vocación para aprender, } \\
\text { menos para enseñar, es muy } \\
\text { difícil..." }\end{array}$ & $\begin{array}{l}\text { que se prestan mucho para práctica a futuro..." } \\
\text { - "....se plantea como premisa intentar convertir } \\
\text { un curso muy teórico en experiencias } \\
\text { vivenciales en donde se les invita a los } \\
\text { participantes a desarrollar actividades y } \\
\text { dinámicas que refuercen y comprendan los } \\
\text { temas vistos, utilizo mucho la resolución de } \\
\text { problemas, comando directo..." }\end{array}$ \\
\hline $\begin{array}{l}\text { “... la teoría no les gusta...” } \\
\text { “... Este es el deseo de todo profesor, } \\
\text { que sus estudiantes estén motivados y } \\
\text { así puedan entender y aprovechar la } \\
\text { información..." } \\
\text { "... la motivación debe ser bilateral..." }\end{array}$ & $\begin{array}{l}\text { “... las estrategias son las correctas, se plantean } \\
\text { con el fin de que el estudiante aprenda..." } \\
\text { “... por medio de las estrategias se busca hacer } \\
\text { un curso teórico más vivencial y llamativo para el } \\
\text { estudiante...” }\end{array}$ \\
\hline
\end{tabular}

Observaciones: en la tabla 5 se presenta la percepción que tienen los docentes de los centros educativos de educación superior participantes en esta investigación, con respecto a las estrategias que usan y su valor en el aprendizaje. Para la categoría motivación se puede observar que los docentes utilizan diferentes estrategias y tienen una visión diferente sobre cómo motivar a los estudiantes, algunos utilizan la práctica de la clase para incentivar a los estudiantes a aprender, otros consideran y se dan cuenta que la teoría no les agrada. A pesar de conocer ciertas estrategias, pareciera que los estudiantes no opinan igual acerca de la motivación que genera el docente en el salón de clase.

Es importante que el docente genere espacios para motivar e incentivar el aprendizaje de sus estudiantes. De acuerdo con Begoña (2005), la motivación es un elemento clave en el proceso de aprendizaje. Si un alumno se encuentra motivado, estará implicado en las

Estrategias pedagógicas utilizadas para la enseñanza de las ciencias del movimiento humano y la percepción estudiantil acerca de su impacto en el aprendizaje

Jorge E. Salas-Cabrera, Carlos Álvarez-Bogantes,

Stephanie Fabiola Ureña-Arias, Oscar José Guillen-Vargas

DOI: http://dx.doi.org/10.22458/caes.v9i2.2147

\section{(c) (i) (5)}


actividades académicas, se concentrará más y, si tiene problemas, él mismo buscará salidas hasta que encuentre posibles alternativas de resolución.

Para la categoría de estrategias pedagógicas, los docentes de ambas modalidades, tanto en lo público como lo privado, consideran que las estrategias utilizadas ayudan de manera permanente al aprendizaje de los estudiantes, sin embargo, al comparar con la percepción que tienen los estudiantes acerca de estas estrategias, parece ser que no se refleja en lo dicho por los docentes, ya que los estudiantes consideran que la clases son muy pasivas, llenas de mucha teoría y poca práctica, siendo esta última la forma que más despierta su atención. Por eso Begoña (2005) determina que si un docente quiere generar motivación del alumnado, lo primero que debe conocer es aquello que quiere, aquellas actividades que despiertan su atención. Según Matos (2005), el objetivo que debe perseguir cada docente en su salón de clase influye en la forma de enseñar, más aún si se tiene en cuenta que él es considerado un importante actor en el proceso de crear estrategias pedagógicas que vayan acorde con la población a la que se dirige.

Tabla 6: Percepciones más importantes acerca de las categorías: retroalimentación y comprensión de contenidos que tienen los docentes de los centros de educación superior públicos y privados en donde se imparte la carrera de Educación Física.

\begin{tabular}{|c|c|}
\hline $\begin{array}{c}\text { CATEGORÍA } \\
\text { RETROALIMENTACIÓN }\end{array}$ & $\begin{array}{c}\text { CATEGORÍA } \\
\text { COMPRENSIÓN DE CONTENIDOS }\end{array}$ \\
\hline \multicolumn{2}{|c|}{ UNIVERSIDADES DEL SECTOR PÚBLICO } \\
\hline $\begin{array}{l}\text { - “...Yo hago una evaluación y esa } \\
\text { me hace saber a mí si quedó todo } \\
\text { entendido, de la mano de las } \\
\text { calificaciones..." } \\
\text { - "...Es acertada en conjunto de las } \\
\text { mismas evaluaciones que yo hago, }\end{array}$ & $\begin{array}{l}\text { - "...Me gusta explicar y que todo quede } \\
\text { entendido pero, lamentablemente, siempre } \\
\text { quedan algunas personas que no ponen } \\
\text { atención y quedan sin entender..." } \\
\text { - "...Es necesaria la evaluación de esta forma, } \\
\text { ambas partes se dan cuenta si el }\end{array}$ \\
\hline
\end{tabular}

Estrategias pedagógicas utilizadas para la enseñanza de las ciencias del movimiento humano y la percepción estudiantil acerca de su impacto en el aprendizaje

Jorge E. Salas-Cabrera, Carlos Álvarez-Bogantes,

Stephanie Fabiola Ureña-Arias, Oscar José Guillen-Vargas

DOI: http://dx.doi.org/10.22458/caes.v9i2.2147

(c) (i) (-)

Artículo protegido por licencia Creative Commons 


\begin{tabular}{|c|c|}
\hline $\begin{array}{l}\text { hay notas muy buenas, muy altas, y } \\
\text { eso me hace ver que el curso y el } \\
\text { aprendizaje van bien..." } \\
\text { - "...Hay que hacer retroalimentación, } \\
\text { si no el ciclo de comunicación } \\
\text { dentro de la educación se pierde..." } \\
\text { - "...Yo siempre ando por ahí y les } \\
\text { pregunto: díganme } \\
\text { aprendieron..." } \\
\text { - “...Yo los trabajos no los entrego } \\
\text { corregidos porque ya sería } \\
\text { demasiado..." } \\
\text {-...El intercambio de información } \\
\text { me gusta que sea mutuo..." } \\
\text { "...los trabajos los devuelvo con } \\
\text { observaciones y si son sobre mal } \\
\text { manejo de conceptos sí se las } \\
\text { aclaro por grupo..." }\end{array}$ & $\begin{array}{l}\text { conocimiento ha sido captado de buena } \\
\text { manera, sin esto no habría educación..." } \\
\text { - "...no saco mucho tiempo para explicar la } \\
\text { práctica porque lo que quiero es que asocien } \\
\text { con la teoría..." }\end{array}$ \\
\hline \multicolumn{2}{|c|}{ EDUCACIÓN SUPERIOR SECTOR PRIVADO } \\
\hline $\begin{array}{l}\text { - “...Cada asignación que ellos } \\
\text { hacen, se les efectúan } \\
\text { observaciones en los trabajos y se } \\
\text { hace una realimentación a nivel } \\
\text { general..." } \\
\text { - “...cuando hay trabajos, cuando } \\
\text { califico exámenes, en fin, siempre } \\
\text { acostumbro hacer una } \\
\text { retroalimentación de la clase...” } \\
\text { - “...en las tareas lo que se hace es } \\
\text { que las respuestas que tienen mal } \\
\text { en los exámenes las tienen que } \\
\text { corregir en la tarea, de forma que } \\
\text { terminan aprendiendo el porqué de } \\
\text { la respuesta equivocada. Y así } \\
\text { identifico las fallas que ellos } \\
\text { tuvieron y cuando me lo entregan lo } \\
\text { reviso y lo entrego de nuevo }\end{array}$ & $\begin{array}{l}\text { - "...la comprensión de los estudiantes creo que } \\
\text { - ha sido buena..." } \\
\text { "...Dentro de la explicación de cada concepto } \\
\text { se les ponen a ellos ejemplos de casos de la } \\
\text { vida real, para transferir conocimiento..." } \\
\text { - "...Las que tienen mayor impacto en el } \\
\text { aprendizaje de los estudiantes son las } \\
\text { vivenciales..." } \\
\text { "...En varios aspectos las estrategias sí son } \\
\text { comprendidas por ellos, pero en ocasiones sí } \\
\text { cuesta que ellos le entiendan a uno qué es lo } \\
\text { que realmente quiere uno que ellos aprendan } \\
\text { del curso..." }\end{array}$ \\
\hline
\end{tabular}

Estrategias pedagógicas utilizadas para la enseñanza de las ciencias del movimiento humano y la percepción estudiantil acerca de su impacto en el aprendizaje

Jorge E. Salas-Cabrera, Carlos Álvarez-Bogantes, 


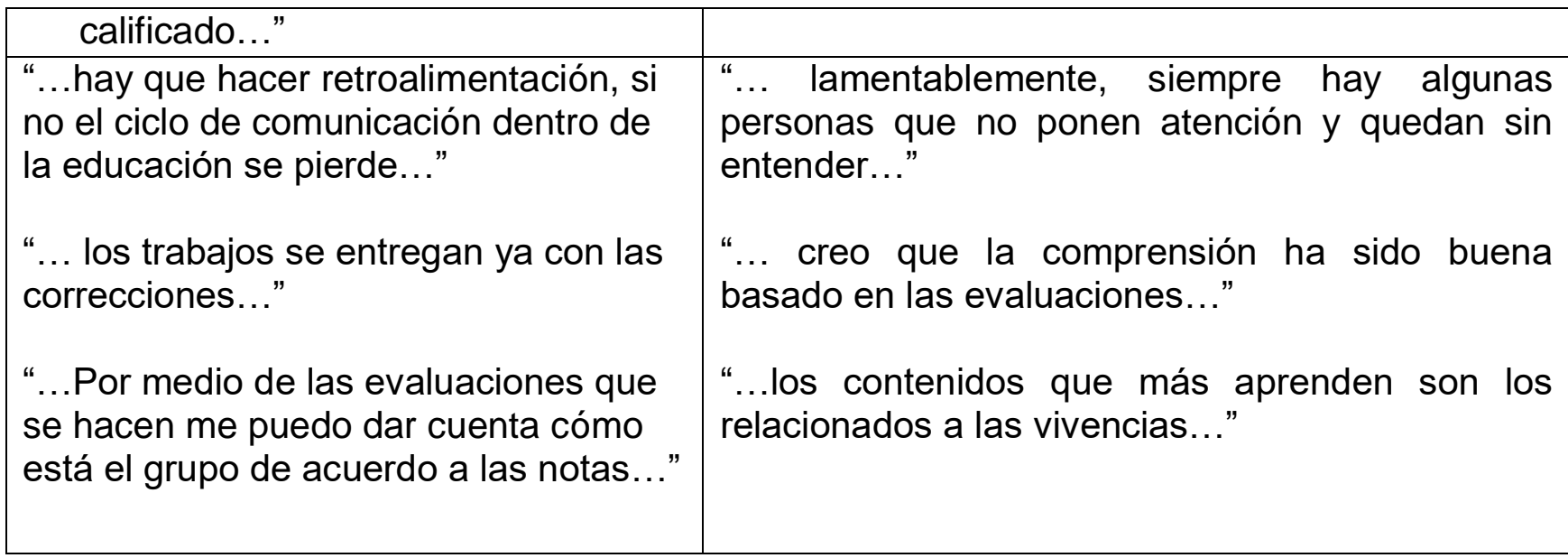

Observaciones: en la tabla 6 se presentan los resultados obtenidos en la entrevista realizada a los 12 docentes que participaron en esta investigación, se extrae aquello que fue reiterativo en las respuestas de las profesoras y los profesores, y lo que fue más significativo para fines de la misma. Para la categoría de retroalimentación, pareciera ser que una parte de los docentes trata, en lo posible, de realizar retroalimentación dentro de su salón de clase, sin embargo, se aprecia que esta es escueta y se muestra poco interés por que todos los estudiantes aprendan en el proceso, en algunos casos ni siquiera se revisan los trabajos y de esta forma es difícil que el estudiante logre aprender. En ese sentido, Valcárcel Cases (2003) explica que se debe ofrecer siempre la retroalimentación necesaria a los estudiantes, sobretodo en relación con las actividades de evaluación formativa.

Para la categoría comprensión de contenidos, los docentes de ambos tipos de educación manifiestan que los estudiantes realmente tienen una comprensión de contenidos significativa, cada uno tiene su forma de realizar la mediación pedagógica, algunos docentes consideran que es con la práctica que los estudiantes realizan, realmente aprenden, sin embargo, esto se contrapone a la percepción del estudiantado, ya que asegura que poca es

Estrategias pedagógicas utilizadas para la enseñanza de las ciencias del movimiento humano y la percepción estudiantil acerca de su impacto en el aprendizaje

Jorge E. Salas-Cabrera, Carlos Álvarez-Bogantes,

Stephanie Fabiola Ureña-Arias, Oscar José Guillen-Vargas

DOI: http://dx.doi.org/10.22458/caes.v9i2.2147

(c) (i) (5)

Artículo protegido por licencia Creative Commons 
la práctica que se realiza y que muchas clases presentan la charla magistral como forma única de enseñanza. Bozu y Canto (2009) consideran que el docente, más que transmitir abundante información, debe promover el desarrollo de competencias como garantía para que los estudiantes puedan seguir aprendiendo a lo largo de su vida y logren desempeñarse de manera pertinente y satisfactoria en un mundo complejo. De ahí, la necesidad de realizar modificaciones en las estrategias pedagógicas de manera que generen no solo un aprendizaje significativo, sino que se despierte en el estudiante ese apetito por querer seguir aprendiendo a lo largo de la vida.

Gráfico 1. Porcentajes alcanzados en las categorías del instrumento "la evaluación del desempeño docente" realizado por estudiantes de bachillerato de la carrera de Educación Física. Universidad pública.

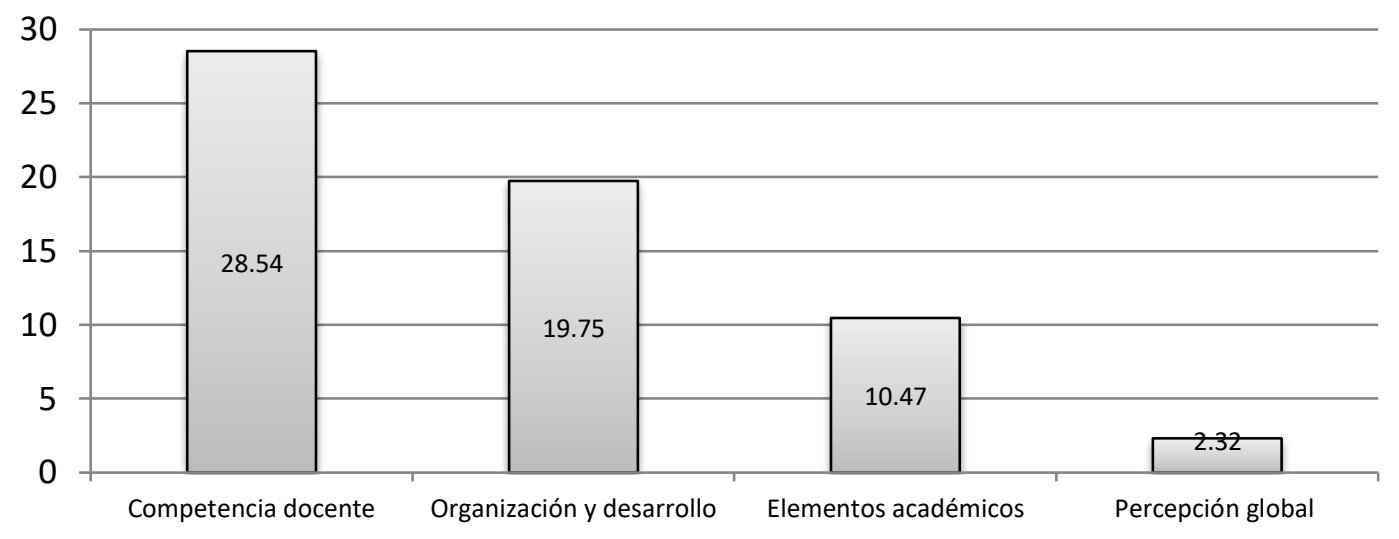

Fuente: Elaboración propia.

Estrategias pedagógicas utilizadas para la enseñanza de las ciencias del movimiento humano y la percepción estudiantil acerca de su impacto en el aprendizaje

Jorge E. Salas-Cabrera, Carlos Álvarez-Bogantes,

Stephanie Fabiola Ureña-Arias, Oscar José Guillen-Vargas

DOI: http://dx.doi.org/10.22458/caes.v9i2.2147

\section{(c) (i) (5)}


Gráfico 2. Porcentajes alcanzados en las categorías del instrumento "la evaluación del desempeño docente" realizado por estudiantes de bachillerato de la carrera de Educación Física. Universidad privada.

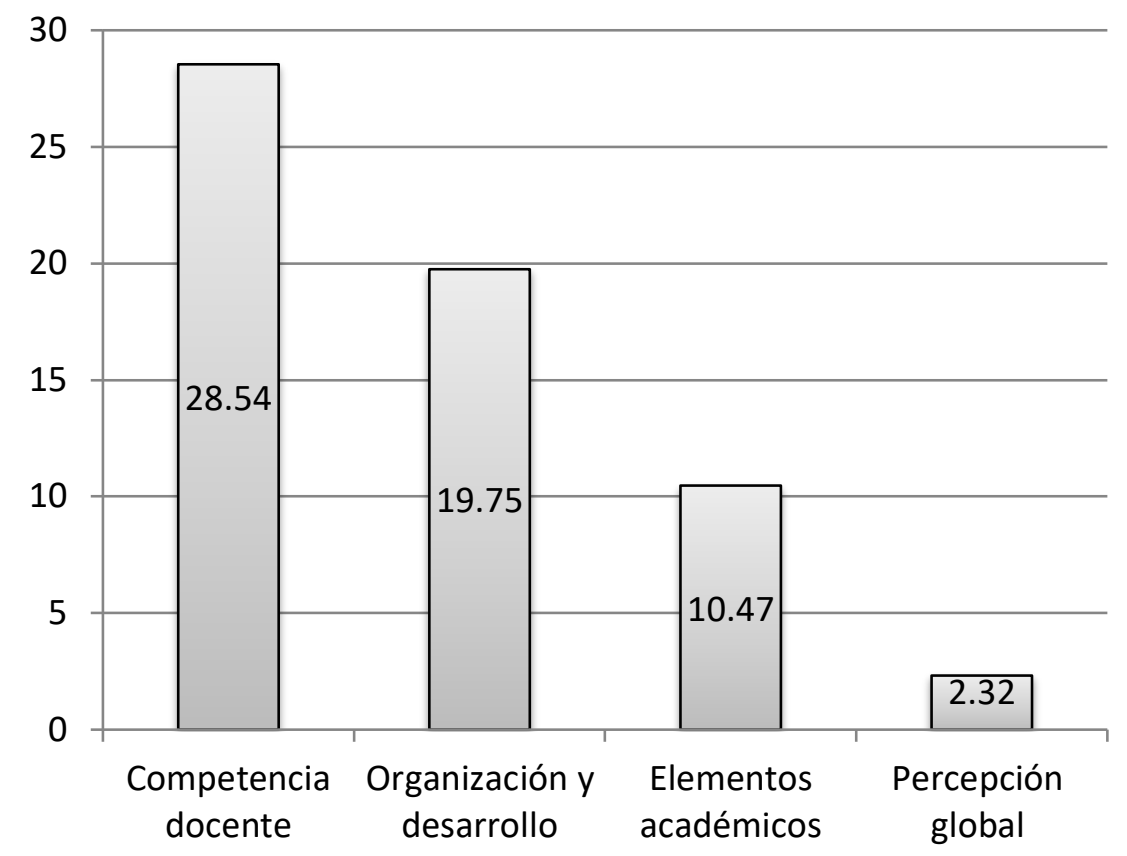

Fuente: Elaboración propia.

\section{Observaciones}

Como se aprecia en los gráficos 1 y 2, los valores representan un máximo de $100 \%$ en cuatro grandes categorías; en las instituciones públicas los estudiantes consideran que el porcentaje que obtienen los docentes es un promedio total de $61,08 \%$ y que los ubica dentro de una categoría regular. Por su parte, los docentes de universidades privadas obtuvieron un $66,09 \%$. Estos gráficos son consecuentes con los resultados obtenidos en los análisis cualitativos, en donde los estudiantes determinan que existen ciertas deficiencias en las

Estrategias pedagógicas utilizadas para la enseñanza de las ciencias del movimiento humano y la percepción estudiantil acerca de su impacto en el aprendizaje

Jorge E. Salas-Cabrera, Carlos Álvarez-Bogantes, 
estrategias pedagógicas utilizadas por los docentes y cómo estas influyen de manera directa en su aprendizaje.

\section{Conclusiones}

Los resultados de esta investigación perciben que existen deficiencias en las estrategias pedagógicas utilizadas por los docentes de educación. Muchos de los estudiantes manifiestan que están cansados de las charlas magistrales y que consideran que su aprendizaje se optimiza en las clases con alto componente práctico. Los docentes generan muy poca motivación para que los estudiantes sigan investigando y preparándose de manera individual, además, pocos de los profesores utilizan la retroalimentación de contenidos como parte del reforzamiento del aprendizaje de contenidos.

\section{Referencias}

Adell, J. (2010). Entornos personales de aprendizaje: Claves para el ecosistema en red. Editorial Marfil. Barcelona, España.

Alterio, G. \& Pérez, H. (2009). Evaluación de la función docente según el desempeño de los profesores y la opinión de los estudiantes. Educación médica Superior, 23(39), 1-14.

Alvarado, J. (2005). Propuesta de estrategias pedagógicas y evaluativas adaptadas al sistema de créditos académicos para la asignatura de inferencia estadística. Bogotá, Colombia: Universidad Javeriana.

Begoña, M. (2005). Adaptación del método docente al Espacio Europeo de Educación Superior: La motivación de los alumnos como instrumento clave. Revista Estudios sobre Educación, 9, 107-126.

Biggs, M. (2005). Calidad del aprendizaje universitario. Editorial Narcea. España.

Boström, L, y Josefsson, G. (2006). Vägar till grammatik. Dinamarca: Narayana Press

Estrategias pedagógicas utilizadas para la enseñanza de las ciencias del movimiento humano y la percepción estudiantil acerca de su impacto en el aprendizaje

Jorge E. Salas-Cabrera, Carlos Álvarez-Bogantes,

Stephanie Fabiola Ureña-Arias, Oscar José Guillen-Vargas

DOI: http://dx.doi.org/10.22458/caes.v9i2.2147

\section{(c) (1) (5)}


Bozu, Z. y Canto, P. J. (2009). El profesorado universitario en la Sociedad del Conocimiento: Competencias Profesionales Docentes. Revista de Formación e Innovación Educativa Universitaria. 2(2), 87-97

Briceño, M.A. (2008). El escrito científico en la universidad. Propuestas de estrategias pedagógicas. Revista Educación y Educadores, 11(2), 107-118.

Craveri, A. \& Anido, M. (2008). El aprendizaje de matemática con herramienta computacional en el marco de la teoría de los estilos de aprendizaje. En Revista de estilos de aprendizaje,1(1), 43-65.

Gargallo, B. (2008). Estilo de docencia y evaluación de los profesores universitarios y su influencia sobre los modos de aprender de sus estudiantes. Revista Española de pedagogía. LXVI (241) 425-445

De Miguel, M. (2005). Cambio de paradigma metodológico en la Educación Superior Exigencias que conlleva. Cuadernos de Integración Europea, 2, 16-27.

Dewey, J. (1997) Demokrati och utbildning. Uddevalla: Mediaprint

Gow, L. y Kember, D. (1993). Conceptions of teaching and their relationship to student learning. British Journal of Educational Psychology, 63, 20-33.

Lee, S. J., Srinivasan, S., Trail, T., Lewis, D. \& Lopez, S. (2011). Examining the relationship among student perception of support, course satisfaction, and learning outcomes in online learning. The internet and higher education. 14(3) 158-163

Marrero, M. y Santana, L. (2016). Estrategia pedagógica para perfeccionar el uso de las imágenes digitales en las Ciencias Médicas. Revista Educación Superior, 30, 60-71.

Martínez, E. (s.f.). El proceso de enseñanza-aprendizaje recuperado en:

http://www.uhu.es/cine.educacion/didactica/0014procesoaprendizaje.htm\#EI proceso de en señanza-aprendizaje

Matos, L. (2005). Desempeño docente y su relación con orientación a la meta, estrategias de aprendizaje y autoeficacia: un estudio con maestros de primaria de Lima, Perú. Revista Universitas Psychologica, 7 (2), 385-401

Estrategias pedagógicas utilizadas para la enseñanza de las ciencias del movimiento humano y la percepción estudiantil acerca de su impacto en el aprendizaje

Jorge E. Salas-Cabrera, Carlos Álvarez-Bogantes,

Stephanie Fabiola Ureña-Arias, Oscar José Guillen-Vargas

DOI: http://dx.doi.org/10.22458/caes.v9i2.2147

\section{(c) (i) (8)}


Molereo, D. (2003). Rendimiento académico y opinión sobre la docencia del alumnado participante en experiencias piloto de implantación del espacio europeo de educación superior. Revista Electrónica de Investigación Evaluativa y Educativa,13(2), 175-190.

Montico, S. (2004). La motivación en el aula universitaria. ¿Una necesidad pedagógica? Revista Ciencia, Docencia y Tecnología, 29, 105-112. Recuperado en: http://www.redalyc.org/pdf/145/14502904 .

Pérez, G. (2001) Investigación cualitativa. Retos e interrogantes. Métodos. Madrid: La Muralla

https://digitum.um.es/jspui/bitstream/10201/17247/1/Adell\%26Casta\%C3\%B1eda 201 $\underline{0 . p d f}$

Ratelle, C. F., Guay, F., Vallerand, R. J., Larose, S. \& Senécal, C. (2007). Autonomous, controlled and amotivated types of academic motivation: A person-oriented analysis. Journal of Educational Psychology, 99(4), 734-746.

Reigeluth, C. M. (1983). Instructional Design: What is it and why is it? En C. M. Reigeluth (Ed.), Instructional-Design Theories and Models: An Overview of their Current Status. Hillsdale: Lawrence Erlbaum

Rivero, C., Díaz, P., Santana, Y. \& Pedraza, Y. (2014). La enseñanza de estrategias de aprendizaje, una perspectiva pedagógica para las transformaciones en la educación superior en Cuba. Revista Pedagogía Universitaria, XIX (2), 16-37.

Standage, M., Duda, J. L. y Ntoumanis, N.A. (2005). Test of selfdetermination theory in school physical education. British Journal of Educational Psychology, 75(3), 411-33. DOI: $10.1348 / 000709904 X 22359$

Tolosa, A. D. \& Zerpa, C. E. (2009). Efecto de un programa de comprensión de textos de contenido moral en las estructuras de razonamiento moral de estudiantes del primer año del Ciclo Diversificado. Revista de Pedagogía, 30(87), 247-280

Torres, A., Ardisana, E., Hernández, G. \& Cabañas, M. (2006). Experiencias educativas de mejoramiento de la calidad centrando las acciones pedagógicas en el aprendizaje: Caso de la fisiología vegetal. Revista Pedagógica Universitaria. VXI (1), 1-9.

Estrategias pedagógicas utilizadas para la enseñanza de las ciencias del movimiento humano y la percepción estudiantil acerca de su impacto en el aprendizaje

Jorge E. Salas-Cabrera, Carlos Álvarez-Bogantes,

Stephanie Fabiola Ureña-Arias, Oscar José Guillen-Vargas

DOI: http://dx.doi.org/10.22458/caes.v9i2.2147

\section{(C) (i) (8)}


Valcárcel, M. (2003). Programa de estudios y análisis destinado a la mejora de la calidad de la enseñanza superior y de la actividad del profesorado universitario. La Preparación del Profesorado Universitario Español para la Convergencia Europea en Educación Superior. Córdoba (España). Revista Educación, 23, 209-213 estudiantil acerca de su impacto en el aprendizaje

Jorge E. Salas-Cabrera, Carlos Álvarez-Bogantes, 